\title{
A DOUBLE BLIND COMPARATIVE STUDY OF CENTCHROMAN WITH EVENING PRIMEROSE OIL IN THE MANAGEMENT OF MASTALGIA IN BENIGN BREAST DISEASE
}

\begin{tabular}{ll}
$\begin{array}{l}\text { Dr. Sandip Kumar } \\
\text { Ghosh }\end{array}$ & $\begin{array}{l}\text { Assoc. Prof, Department of General Surgery, Burdwan Medical College and } \\
\text { Hospital. }\end{array}$ \\
\hline $\begin{array}{l}\text { Dr. Sukhamoy } \\
\text { Saha* }\end{array}$ & $\begin{array}{l}\text { Assist. Prof, Dept. of General Surgery, Burdwan Medical College and } \\
\text { Hospital. *Corresponding Author }\end{array}$ \\
\hline Dr. Chayan Gupta & MS, General Surgery ( Burdwan Medical College and Hospital). \\
\hline Dr. Prabir Biswas & $3^{\text {rd }}$ Year PGT, MS General Surgery, Burdwan Medical College and Hospital. \\
\hline \hline
\end{tabular}

ABSTRACT INTRODUCTION-Pain is the most common reason for referral to a breast clinic and accounts for up to 50 per cent of patients seen. It is, however, the least understood of all breast symptoms, and the one whose management causes the most controversy. It must be stressed that mastalgia is a symptom and does not imply any specific pathological process. There is considerable debate about drug of choice for management of mastalgia. We present our results of a trial of antiestrogen drug "Centchroman". The objective of the study was to evaluate the effectiveness of Centchroman in control of mastalgia measured by visual analogue scale (VAS), and compare it with that of Danazol. OBJECTIVES- To evaluate the efficacy, side effect, cost effectiveness of Centchhroman versus Danazol and Evening Primrose Oil in regression of mastalgia in benign breast diseases. PROCEDURE-The study was carried out on 105 patients with mastalgia diagnosed as benign breast disease with VAS score $\geq 3$ lasting for more than 7 days per cycle are included in this study and divided into 3 groups and the study conducted. CONCLUSION- From this study it can be concluded that Centchroman is very cost effective , efficacious with minimal side effect for treatment of mastalgia in beningn breast disease. Although a longer follow up period is necessary to prove its long term efficacy.

\section{KEYWORDS : Mastalgia, Centchroman, Danazol, Evening Prime Rose Oil, Vas}

\section{INTRODUCTION}

Pain is the most common reason for referral to a breast clinic and accounts for up to 50 per cent of patients seen. It is, however, the least understood of all breast symptoms, and the one whose management causes the most controversy.

Reports on breast pain are often anecdotal, uncontrolled, and of poor quality.

Mastalgia has also been correlated with specific histological criteria, resulting in its description as 'fibrocystic disease', although this has lost favour for reasons described above.

It must be stressed that mastalgia is a symptom and does not imply any specific pathological process. The pattern and severity of pain can be assessed by breast pain chart. Mastalgia may be cyclic or non-cyclic, intermittent or constant, localized or diffuse. There are two distinct group of patients with these symptoms :

one group has symptoms that bear a definite relation to the menstrual cycle (cyclical mastalgia); in the remainder there is no such correlation (non-cyclical mastalgia).

Many pharmacological agents have been tried in the therapy of mastalgia. The drug therapy includes agents inducing hormonal manipulation such as Danazol, Bromocriptine, Tamoxifen, and LH-RH analogue like Goserelin. Some of the effective non-hormonal agents in mastalgia are Nonsteroidal anti- inflammatory gels, reassurance and breast support with sport's bra.

There is considerable debate about drug of choice for management of mastalgia. We present our results of a trial of antiestrogen drug "Centchroman". The objective of the study was to evaluate the effectiveness of Centchroman in control of mastalgia measured by visual analogue scale (VAS), and compare it with that of Danazol.

Research hypothesis: Centchroman therapy offers a safe, at least equally or more effective and inexpensive alternative to Danazol and evening primrose oil for treatment of mastalgia.

AIM of the study-

1. To evaluate the efficacy of Centchroman versus Danazol and Evening Primrose Oil in regression of mastalgia in benign breast diseases.

2. To compare the side effect profile of Centchroman versus Danazol and Evening Primrose Oil in the treatment of mastalgia.

3. To compare the cost of treatment with Centchroman versus Danazol and Evening Primrose Oil in the treatment of mastalgia.

\section{PROCEDURE-}

The study was carried out on 105 patients attended to the outdoor, dept. of General Surgery, Burdwan Medical College and Hospital with complain of mastalgia during the period January 2018- January 2019. All premenopausal women with mastalgia diagnosed as benign breast disease with VAS score $\geq 3$ lasting for more than 7 days per cycle are included in this study. All patients with malignant breast disease, dysplastic lesion which mandate compulsory surgical intervention, simple breast cysts, patients with clinically palpable or ultrasonographically detectable lump $>5 \mathrm{~cm}$ in size, patients on oral contraceptive pills, patients desirous to remove the lump, patients unwilling to enrol herself in the study, past history of breast carcinoma or family history of breast carcinoma and patients in their first six months of Lactation are excluded. Patients having breast pain are screened and 105 patients are selected meeting the inclusion criteria allocated into 3 groups namely $A, B, C$ of 35 patients in each group. Before initiation of the study prior informed consent has been obtained. Further necessary investigations were done to exclude other possibilities. Cases of mild mastalgia treated with supportive care and not included in the study. All the patients received the same supportive care that included reassurance, breast support and stopping of xanthenes in diet (abstinence from coffee, tea etc.). Patients given Danazol $100 \mathrm{mg} /$ day, Centchroman $30 \mathrm{mg} /$ day and 
Evening Primrose Oil $3 \mathrm{gm} /$ day for a period of 12 weeks and then followed for 12 weeks after stopping drug for sustenance of response and recurrence. The 3 drugs are packed in 3 separate packages by a Nursing Sister and the packages will be ascribed with letter $a, b, c$ which will be blinded to the investigator as well as the patients treated with Evening Primrose Oil , the second arm with Danazol and the third with Centchroman. All the cases are recorded in a predesigned and pretested schedule. Patients were asked to keep a record of their breast pain in a 'pain diary'. In this diary patient filled the occurrence of pain on a day to day basis. The time of menses was also marked on pain diary. The severity of mastalgia was assessed by visual Analogue scale score ranging from $0-10$.

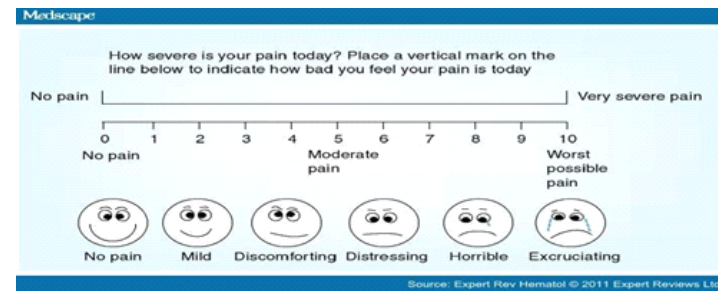

Visual analogue scale

RESULT AND ANALYSIS

1.Association with age -In group-A, 13(37.1\%) patients had $<30$ years of age, $20(57.1 \%)$ patients had $30-39$ years of age and $2(5.7 \%)$ patients had 40 years of age. In group-B, $15(42.9 \%)$ patients had $<30$ years of age, $19(54.3 \%)$ patients had $30-39$ years of age and $1(2.9 \%)$ patient had 40 years of age. In group-C, $10(28.6 \%)$ patients had $<30$ years of age, $23(65.7 \%)$ patients had $30-39$ years of age and $2(5.7 \%)$ patients had 40 years of age. Association of age vs. group was not statistically significant $(p=0.7689)$.

2.Association with type of mastalgia-In group-A, 29(82.9\%) patients had Non cyclical and 6(17.1\%) patients had cyclical. In group-B, 27(77.1\%) patients had Non cyclical and $8(22.9 \%)$ patients had cyclical. In group-C, $21(60.0 \%)$ patients had Non cyclical and 14(40.0\%) patients had cyclical. Association of type of mastalgia vs. group was not statistically significant $(\mathrm{p}=0.0795)$.

3.Āssociation between VAS at presentation-GROUP

\begin{tabular}{|c|c|c|c|c|}
\hline \multicolumn{5}{|c|}{ GROUP } \\
\hline VAS at presentation & Group-A & Group-B & Group-C & TOTAL \\
\hline 4 & 12 & 11 & 8 & 31 \\
\hline Row \% & 38.7 & 35.5 & 25.8 & 100.0 \\
\hline Col \% & 34.3 & 31.4 & 22.9 & 29.5 \\
\hline 5 & 6 & 5 & 6 & 17 \\
\hline Row \% & 35.3 & 29.4 & 35.3 & 100.0 \\
\hline Col \% & 17.1 & 14.3 & 17.1 & 16.2 \\
\hline 6 & 6 & 7 & 8 & 21 \\
\hline Row \% & 28.6 & 33.3 & 38.1 & 100.0 \\
\hline Col \% & 17.1 & 20.0 & 22.9 & 20.0 \\
\hline 7 & 1 & 2 & 4 & 7 \\
\hline Row \% & 14.3 & 28.6 & 57.1 & 100.0 \\
\hline Col \% & 2.9 & 5.7 & 11.4 & 6.7 \\
\hline 8 & 10 & 10 & 9 & 29 \\
\hline Row \% & 34.5 & 34.5 & 31.0 & 100.0 \\
\hline Col \% & 28.6 & 28.6 & 25.7 & 27.6 \\
\hline TOTAL & 35 & 35 & 35 & 105 \\
\hline Row \% & 33.3 & 33.3 & 33.3 & 100.0 \\
\hline Col \% & 100.0 & 100.0 & 100.0 & 100.0 \\
\hline
\end{tabular}

Association of VAS at presentation vs. group was not statistically significant $(p=09134)$

4.Distribution of mean Age vs. Group

In group $-\bar{A}$, the mean age (mean \pm s.d.) of patients was 31.1429 \pm 5.2195 years. In group-B, the mean age (mean \pm s.d.) of patients was $30.6286 \pm 5.4129$ years. In group-C, the mean age (mean \pm s.d.) of patients was $32.2000 \pm 5.6141$ years. Distribution of mean age vs. group was not statistically significant $(p=0.4678)$.

5.Asssociation between VAS at 4,8,12 weeks vs. Group Association of VAS at 4,8,12 weeks vs. group was statistically significant, each have a p value $<0.001$.

6.Association between Recurrence of pain in next 12 weeks follow up vs. Group

In group- $A, 2(5.7 \%)$ patients had recurrence of pain in next 12 weeks follow up. In group-B, $7(20.0 \%)$ patients had recurrence of pain in next 12 weeks follow up. In group-C, 32(92.4\%) patients had recurrence of pain in next 12 weeks follow up. Association of recurrence of pain in next 12 weeks follow up vs. group was statistically significant $(\mathrm{p}<0.001)$

\section{Association between Side effect vs. Group}

In group- $\mathrm{A}, 2(5.7 \%)$ patients had delayed menses, $1(2.9 \%)$ patient had missed period and $32(91.4 \%)$ patients side effects were not applicable. In group-B, $1(2.9 \%)$ patient had irregular menses, 2(5.7\%) patient had missed period and $32(91.4 \%)$ patients' side effects were not applicable. In group-C, $35(100.0 \%)$ patients' side effects were not applicable. Association of side effects vs. group was not statistically significant $(p=0.2251)$.

8.Association between Cost of treatment for 84 days vs. Group

There is a huge difference in the cost of treatment for the 3 drugs for a course of 84 days. Whereas Centchroman costs 3 Rs. / tablet it costs 252 Rs. For a course of 84 days , Danazol 100 mg capsules costs 27 Rs. Per capsule and likewise costs around 4536 Rs. For a 84 days course the differencebetween cost of treatment between the two drugs is significant with $\mathrm{p}$ value <.05. EPO with a $3 \mathrm{gm} /$ day dose costs almost double that of Danazol of around 7560 Rs. For a 84 day course and is significantly higher than that of centchroman

\section{DISCUSSION}

Severe mastalgia or breast pain interfere with daily routine activites of many woman of reproductive age group and moreover raises fear of having underlying cancer. In many patients reassurance, tight brassiere or sports bra often suffice. A Brazilian study showed success rate of around $70.2 \%$ with reassurance alone in a study of 85 patients with mastalgia. Other non medical measures like dietary fat restriction and avoidance of methylxanthines lik tea or coffee. In our study baseline mean VAS was 5.7429 in Centchroman group that is Group A , 5.8571 in Danazol Group that is Group $\mathrm{B}, 6.0000$ in Evening primrose oil group that is Group C.

A. In Group B or danazol group mean VAS change at 4 wks , 8 wks and 12 wks was $3.2571,2.8000,1.8571$ which is statistically significant $(\mathrm{p}<.001)$. Our study shows similar findings as the study of Greenblatt et al. which noticed alleviation of breast pain , nodularity and premenstrual breast engorgement with the administration of danazol in management of endometriosis and further on study he experienced that danazol is an excellent drug in management of mastalgia and nodularity in $\sim 75 \%$ of women. In a metaanalysis of 4 randomized trials they found a highly significant relief of mastalgia using Danazol, as compared to placebo.

B. In our study patients in Group A (centchroman ) and Group B (Danazol ) showed gradual improvement of symptoms wheres improvement in Group C (Evening primrose oil ) was unremarkable. Mean VAS in 3 groups at the end of 12 weeks of treatment was $1.1714,1.2161,3.4571$ respectively in the three 
groups. The difference in VAS in three groups was statistically significant with a p value of $<.001$. Tejwani et al in 2011 in a similar study comparing danazol and Centchroman only reported relief of breast pain in $89.7 \%$ as compared to $69.44 \%$ in group B at 12 weeks of treatment. In study conducted by Dhar et al in 2007 and Kumar S et al in 2013 to see the effect of centchroman in mastalgia, there was $100 \%$ and $93.3 \%$ improvement in mastalgia at the end of 3 months and decrease in mean VAS to 0.00 and 1.39 respectively. These results are almost similar to our study.

C. In our study, baseline mean VAS was $6 \pm 1.5146$ in EPO group. In Evening Primrose Oil group after starting the treatment mean VAS was $4.8857 \pm 1.1317$ at 4 wks, $4.3429 \pm$ 1.0556 at 8 wks and $3.4571 \pm 1.3138$ at 12 wks and after stopping Evening Primrose Oil for 3 months VAS at 12 weeks of followup $91.5 \%$ of patients showed recurrence of pain with a VAS $>3$. From study it is evident that there is reduction of (VAS) present after starting of Evening Primrose Oil though much less than the other 2 groups in between 0 and at 12 wks indicating good response of drugs in decreasing breast pain . But there was significant recurrence in pain (VAS) during follow up period of 3 months after stopping of drug, which indicate that Evening Primrose Oil has no long term efficacy for treatment of mastalgia.

In our study there was significant reduction of pain (VAS) present after starting of Danazol in between 0 and at $4 \mathrm{wks}$ ,8wks, and 12 wks $(p<0.05)$ indicating good response of Danazol in treatment of mastalgia. But there was significant increase in VAS during the next 12 weeks of follow up after stopping of Danazol with recurrence of pain (VAS >3) in $20 \%$. Danazol has no or less long term efficacy in treatment of mastalgia. Recently published meta-analysis of randomized placebo controlled clinical trial by Srivastava A et al. in 2007 on drug treatment of mastalgia included 4 common agents viz, Bromocriptine, Tamoxifen, Evening Primrose Oil (EPO) and Danazol. Weighted mean difference in pain score in favour of Bromocriptine was- 16.31 (95\% CI-26.35 to -6.27), Danazol had benefit mean pain score difference 20.23 (95\% CI-28.12 to -12.34), EPO didn't show any advantage with mean pain score-2.78(95\%CI-7.97 to -2.40) and Tamoxifen achieved a relative risk (RR) of pain relief of 1.92 (95\% CI-1.42 to -2.58) with least side effects and was recommended to be the drug of first choice for treating mastalgia by the authors.

D. In our study there is dramatic reduction in mean VAS after starting of Centchroman in between 0 and 4 wks , 8 wks , 12 wks indicating good response of Centchroman. On stopping treatment at $12 \mathrm{wks}$, these patients followed up for another 12 wks. There was no significant increase in pain (VAS) during the follow up for a period odf 12 wks with only $5.7 \%$ of patients showed recurrence ( VAS >3). This indicates long term efficacy of Centchroman after discontinuation of drug and patients remain pain free. At 12 wks of treatment mean VAS was $1.1714 \pm 1.0142$ and $82.8 \%$ of patients had VAS $<3$, $25.71 \%$ patients had VAS $=0$ or no pain. At 12 wks follow up after stopping the drug for 12 wks 2 patient showed recurrence of pain i. e. VAS $>3$ or $94.28 \%$ patients had VAS persistently lower than 3 , in other words showed continued response even after drug withdrawal.

E. In our study, at 12 weeks Centchroman achieved $94.28 \%$ response rate (reduction of pain to less than or equal to 3 on VAS), Danazol achieved $91.42 \%$ response rate and EPO achieved $51.42 \%$ response rate. At 24 weeks, Centchroman achieved $94.3 \%$ response rate, Danazol achieved $80 \%$ response rate and EPO achieved $8.6 \%$ response rate. In our study, both Centchroman and Danazol are efficacious at 12 wks of treatment. reproductive age group in $2^{\text {nd }}$ and $3^{\text {rd }}$ deacde of life.

2. Centchroman is more efficacious than Danazol and Evening Primrose Oil in rapid and sustained pain relief in Mastalgia .

3. Danazol also provide good pain relief in mastalgia patients but has recurrence of pain after stopping treatment .

4. Evening Primrose Oil provide some pain relief in both cyclical and non cyclical mastalgia though it is insignificant and response is unpredictable.

5. The efficacy of Danazol is much more in mastalgia than Evening Primrose Oil in term of pain relief.

6. Centchroman is minimally associated with some acceptable side effects, whether Danazol is also associated fewer adverse effects in $100 \mathrm{mg}$ bid dose and Evening Primrose Oil is associated with almost no adverse effects.

7. Very high cost of Danazol and Evening Primrose Oil can render these regimens as less preferable over Centchroman which has a much wider safety profile and minimum contraindications making it suitable for use in a larger population of patients with mastalgia.

\section{CONCLUSION}

1. Mastalgia is a condition affecting females mostly in their 